\title{
Extinction of quasiparticle scattering interference in cuprate superconductors
}

\author{
Zhi Wang, Bin Liu, and Shiping Feng* \\ Department of Physics, Beijing Normal University, Beijing 100875, China
}

\begin{abstract}
The quasiparticle scattering interference phenomenon characterized by the peaks in the local density of states is studied within the kinetic energy driven superconducting mechanism in the presence of a single impurity. By calculation of the Fourier transformed ratio of the local density of states at opposite energy, it is shown that the quasiparticle scattering interference phenomenon can be described qualitatively by a single impurity in the kinetic energy driven homogeneous dwave superconducting state. The amplitude of the peak increases with increasing energy at the low energy, and reaches a maximum at the intermediate energy, then diminishes to zero at the high energy. The theory also predicts that with increasing doping, the position of the peak along the nodal direction moves towards to the center of the Brillouin zone, while the position of the peak along the antinodal direction is shifted to large momentum region.

PACS numbers: 74.20.-z, 74.25.Jb, 74.50.+r, 74.72.-h

Keywords: Quasiparticle scattering interference; Local density of states; Cuprate superconductors; Kinetic energy driven superconducting mechanism
\end{abstract}

In the study of the physical properties of cuprate superconductors, one of powerful techniques is scanning tunneling microscopy (STM) $\frac{2}{2}$, since it is the only method to probe the real-space inhomogeneous electronic structure of cuprate superconductors. Application of STM to cuprate superconductors would allow one to explore some important issues ${ }^{2-\underline{5}}$, including (1) the physical processes dominating quasiparticle scattering, (2) the degree to which the quasiparticles are well defined and coherent, and (3) the relation between the commensurate and incommensurate magnetic signatures and the quasiparticle scattering processes. In particular, STM has been used to infer the momentum-space information of quasiparticles from the Fourier transform of the position $(\mathbf{r})$ and energy $(\omega)$-dependent local density of states (LDOS), $\rho(\mathbf{r}, \omega)$, then both real-space and momentum-space modulations for LDOS are explored simultaneously ${ }^{4}$. The typical feature observed by the Fourier transformed LDOS is dominated by the peaks at well-defined wavevectors $\mathbf{q}_{i}$ obeying the octet mode ${ }^{3-\underline{5}}$, since the quasiparticle dispersion in cuprate superconductors in the superconducting (SC) state has closed constant-energy contours surrounding the d-wave nodes. Theoretically, there is a general consensus that the peak emerges due to the quasiparticle scattering interference (QSI) $\underline{\underline{6}-8,10}$. This QSI is manifested itself as spatial modulation in the Fourier transformed LDOS $\rho(\mathbf{q}, \omega)$ whose wavevectors change with energy. Several attempts have been made to make this argument more precise. In particular, some calculations based on the phenomenological d-wave Bardeen-CooperSchrieffer (BCS) formalism have been performed by considering the effect of impurity scattering $\underline{\underline{6}} \underline{-11}$, where it has been shown that a single or few impurities in a homogeneous d-wave SC state leads to a result that is in qualitative agreement with the STM experimental data ${ }^{3-5}$.

Recently, the improvements in the resolution of STM experiments $\frac{12-14}{14}$ allowed one to resolve additional features in QSI. Among these new achievements is the mea-

*Corresponding author. Tel.: +86 10 58806408; Fax: +86 10 58801764; E-mail address: spfeng@bnu.edu.cn surement of the ratio of differential conductances at opposite bias,

$$
Z(\mathbf{r}, V) \equiv g(\mathbf{r},+V) / g(\mathbf{r},-V),
$$

where $V$ is the bias voltage and $g(\mathbf{r}, V)$ the differential conductance. The advantage of this procedure is that it cancels the severe systematic errors in the measurement of $g(\mathbf{r},+V)$ due to tip elevation errors ${ }^{12-14}$, yet retains all the quasiparticle information in the differential conductance. In particular, an remarkable phenomenon observed from these new STM experiments is that QSI always disappears at a scale energy (high energy) that is indistinguishable from the energy at which electronic homogeneity is lost. In other words, at energies below the high energy, the STM response is similar to the dispersing Bogoliubov quasiparticle, however, at energies above the high energy, the response becomes highly spatially inhomogeneous. In corresponding to the constant-energy contour of the high energy, an extinction line exists $\frac{14}{}$ in momentum space [near the diagonal line between $( \pm \pi, 0)$ and $(0, \pm \pi)$ ], beyond which most of the dispersing peaks disappear, to be replaced by a reduced set of roughly nondispersive peaks. These new STM experimental results lead naturally to speculation about the appearance of the QSI extinction in cuprate superconductors in the SC state near the boundary of the Brillouin zone (antinodal regime). Within the phenomenological BCS formalism, the QSI phenomenon in a d-wave SC state with coexisting short-range antiferromagnetic (AF) order has been studied ${ }^{15,16}$ recently by considering the scattering arising from a single point-like impurity, and the result shows that dispersing peaks are then extinguished at the high energy $1 \frac{15}{}$. To the best of our knowledge, the dispersing peaks below the high energy in cuprate superconductors and its extinction at the high energy has not been treated starting from a microscopic SC theory, and no explicit calculations of the doping dependence of the dispersing peaks has been made so far. In this paper we show very clearly if the effect of a single point-like impurity scattering potential is considered within the kinetic energy driven SC mechanism 17 , the calculated ratio of LDOS at opposite energy in momentum-space can reproduce some main features observed experimentally on 
cuprate superconductors $4,5,14$, including the appearance of the dispersing peaks below the high energy and its extinction at the high energy. With increasing doping concentration, the position of the peak along the nodal direction moves towards to the center of the Brillouin zone, while the position of the peak along the antinodal direction is shifted to large momentum region.

In cuprate superconductors, the single common feature is the presence of the two-dimensional $\mathrm{CuO}_{2}$ plane, and then it is believed that the unconventional physical properties of cuprate superconductors is closely related to the doped $\mathrm{CuO}_{2}$ planes 18 . It has been argued that the $t-J$ model on a square lattice,

$$
\begin{aligned}
H & =-t \sum_{i \hat{\eta} \sigma} C_{i \sigma}^{\dagger} C_{i+\hat{\eta} \sigma}+t^{\prime} \sum_{i \hat{\tau} \sigma} C_{i \sigma}^{\dagger} C_{i+\hat{\tau} \sigma}+\mu \sum_{i \sigma} C_{i \sigma}^{\dagger} C_{i \sigma} \\
& +J \sum_{i \hat{\eta}} \mathbf{S}_{i} \cdot \mathbf{S}_{i+\hat{\eta}}
\end{aligned}
$$

acting on the Hilbert subspace with no double occupied site, i.e., $\sum_{\sigma} C_{i \sigma}^{\dagger} C_{i \sigma} \leq 1$, captures the essential physics of the doped $\mathrm{CuO}_{2}$ plane $\frac{19}{}$, where $\hat{\eta}= \pm \hat{x}, \pm \hat{y}, \hat{\tau}= \pm \hat{x} \pm \hat{y}$, $C_{i \sigma}^{\dagger}\left(C_{i \sigma}\right)$ is the electron creation (annihilation) operator, $\mathbf{S}_{i}=\left(S_{i}^{x}, S_{i}^{y}, S_{i}^{z}\right)$ are spin operators, and $\mu$ is the chemical potential. To deal with the constraint of no double occupancy in analytical calculations, the charge-spin separation (CSS) fermion-spin theory ${ }^{20}$ has been developed, where the constrained electron operators are decoupled as $C_{i \uparrow}=h_{i \uparrow}^{\dagger} S_{i}^{-}$and $C_{i \downarrow}=h_{i \downarrow}^{\dagger} S_{i}^{+}$, with the spinful fermion operator $h_{i \sigma}=e^{-i \Phi_{i \sigma}} h_{i}$ describes the charge degree of freedom together with some effects of the spin configuration rearrangements due to the presence of the doped hole itself (charge carrier), while the spin operator $S_{i}$ describes the spin degree of freedom (spin), then the electron local constraint for single occupancy, $\sum_{\sigma} C_{i \sigma}^{\dagger} C_{i \sigma}=S_{i}^{+} h_{i \uparrow} h_{i \uparrow}^{\dagger} S_{i}^{-}+S_{i}^{-} h_{i \downarrow} h_{i \downarrow}^{\dagger} S_{i}^{+}=$ $h_{i} h_{i}^{\dagger}\left(S_{i}^{+} S_{i}^{-}+S_{i}^{-} S_{i}^{+}\right)=1-h_{i}^{\dagger} h_{i} \leq 1$, is satisfied in analytical calculations. In particular, it has been shown ${ }^{21}$ that under the decoupling scheme, this CSS fermionspin representation is a natural representation of the constrained electron defined in the Hilbert subspace without double electron occupancy. Furthermore, these charge carrier and spin are gauge invariant ${ }^{20.21}$, and in this sense they are real and can be interpreted as physical excitations 22 . This is much different from the usual slaveboson approach ${ }^{23.24}$, where the electron operator is decomposed as the holon and spinon, however, the local constraint for the single occupancy is explicitly replaced by a global constraint in the mean-field level. Due to the constraint, these holon and spinon are also coupled by a strong gauge field ${ }^{23,24}$, allowed by this slave-boson representation, and therefore these holon and spinon are not gauge invariant. From this point of view, our treatment of constraint for the physical electron may be better than the usual slave-boson approach. In this CSS fermion-spin representation, the $t-J$ Hamiltonian (2) can be expressed as,

$$
\begin{aligned}
H & =t \sum_{i \hat{\eta}}\left(h_{i+\hat{\eta} \uparrow}^{\dagger} h_{i \uparrow} S_{i}^{+} S_{i+\hat{\eta}}^{-}+h_{i+\hat{\eta} \downarrow}^{\dagger} h_{i \downarrow} S_{i}^{-} S_{i+\hat{\eta}}^{+}\right) \\
& -t^{\prime} \sum_{i \hat{\tau}}\left(h_{i+\hat{\tau} \uparrow}^{\dagger} h_{i \uparrow} S_{i}^{+} S_{i+\hat{\tau}}^{-}+h_{i+\hat{\tau} \downarrow}^{\dagger} h_{i \downarrow} S_{i}^{-} S_{i+\hat{\tau}}^{+}\right) \\
& -\mu \sum_{i \sigma} h_{i \sigma}^{\dagger} h_{i \sigma}+J_{\mathrm{eff}} \sum_{i \hat{\eta}} \mathbf{S}_{i} \cdot \mathbf{S}_{i+\hat{\eta}},
\end{aligned}
$$

with $J_{\text {eff }}=(1-\delta)^{2} J$, and $\delta=\left\langle h_{i \sigma}^{\dagger} h_{i \sigma}\right\rangle=\left\langle h_{i}^{\dagger} h_{i}\right\rangle$ is the hole doping concentration. As a consequence, the kinetic energy term in the $t-J$ model has been transferred as an interaction between charge carriers and spins, which reflects that even the kinetic energy term in the $t-J$ Hamiltonian has a strong Coulombic contribution due to the restriction of no double electron occupancy of a given site.

For the understanding of the physical properties of cuprate superconductors in the SC state, we have developed a kinetic energy driven $\mathrm{SC}$ mechanism $\frac{17}{\underline{1}}$, where the interaction between charge carriers and spins arising directly from the kinetic energy term in the $t$ - $J$ model (3) induces a d-wave charge carrier pairing state by exchanging spin excitations in the higher power of the hole doping concentration, then the electron Cooper pairs originating from the charge carrier pairing state are due to the charge-spin recombination, and their condensation reveals the SC ground-state. In particular, this d-wave SC state is controlled by both the SC gap function and the quasiparticle coherent weight, which leads to a fact that the maximal SC transition temperature occurs around the optimal doping, and then decreases in both underdoped and overdoped regimes. Furthermore, it has been shown that this SC state is a conventional BCS-like with the d-wave symmetry 25 , so that the basic BCS formalism with a d-wave SC gap function is still valid in quantitatively reproducing some main low energy features of the SC coherence of quasiparticles, although the pairing mechanism is driven by the kinetic energy by exchanging spin excitations. Within this kinetic energy driven superconductivity, we have discussed the low energy electronic structure ${ }^{21.25}$ of cuprate superconductors, the dynamical spin response $\frac{17.26}{}$, and the quasiparticle transport in the $\mathrm{SC}$ state $\frac{27}{2}$, and qualitatively reproduced some main features of ARPES experiments 18 , inelastic neutron scattering experiments ${ }^{28,29}$, and microwave conductivity measurements $\frac{30}{30}$ on cuprate superconductors. The typical feature of this kinetic energy driven SC mechanism is that the pairing comes out from the kinetic energy by exchanging spin excitations and is not driven by the magnetic superexchange interaction as in the resonant valence bond type theories 19 . In particular, a possible SC theory has been developed for description of superconductivity in doped hexaborides 31 , where the physical mechanism favoring such a reorientation is the enhanced coherence (and hence lower kinetic energy) of the doped electrons in a ferromagnetic background relative to the paramagnet. Following the previous discussions $\frac{17,25}{}$, the charge carrier diagonal and off-diagonal Green's func- 
tions of the $t$ - $J$ model (3) can be obtained as,

$$
\begin{aligned}
& g_{11}(\mathbf{k}, \omega)=Z_{h F}\left(\frac{U_{h \mathbf{k}}^{2}}{\omega-E_{h \mathbf{k}}}+\frac{V_{h \mathbf{k}}^{2}}{\omega+E_{h \mathbf{k}}}\right) \\
& g_{21}(\mathbf{k}, \omega)=-Z_{h F} \frac{\bar{\Delta}_{h Z}(\mathbf{k})}{2 E_{h \mathbf{k}}}\left(\frac{1}{\omega-E_{h \mathbf{k}}}-\frac{1}{\omega+E_{h \mathbf{k}}}\right)
\end{aligned}
$$

where the charge carrier quasiparticle spectrum $E_{h \mathbf{k}}=$ $\sqrt{\bar{\xi}_{\mathbf{k}}^{2}+\left|\bar{\Delta}_{h Z}(\mathbf{k})\right|^{2}}$ with the renormalized d-wave charge carrier pair gap function $\bar{\Delta}_{h Z}(\mathbf{k})=\bar{\Delta}_{h Z}\left[\cos k_{x}-\cos k_{y}\right] / 2$, and the charge carrier quasiparticle coherence factors $U_{h \mathbf{k}}^{2}=\left(1+\overline{\xi_{\mathbf{k}}} / E_{h \mathbf{k}}\right) / 2$ and $V_{h \mathbf{k}}^{2}=\left(1-\overline{\xi_{\mathbf{k}}} / E_{h \mathbf{k}}\right) / 2$, while the charge carrier quasiparticle coherent weight $Z_{h F}$ and other notations are defined as same as in Ref. 25, and have been determined by the self-consistent calculation 17,25 . For the convenience of the following discussions, the full charge carrier Green functions (4) and (55) can also be expressed in the Nambu representation as,

$$
g(\mathbf{k}, \omega)=Z_{\mathrm{hF}} \frac{\omega \tau_{0}+\bar{\xi}_{\mathbf{k}} \tau_{3}-\bar{\Delta}_{\mathrm{hZ}}(\mathbf{k}) \tau_{1}}{\omega^{2}-E_{\mathrm{hk}}^{2}}
$$

where $\tau_{0}$ is the unit matrix, $\tau_{1}$ and $\tau_{3}$ are Pauli matrices.

In the CSS fermion-spin representation ${ }^{20.21}$, the electron Green's function in the Nambu representation,

$$
G(\mathbf{k}, \omega)=\left(\begin{array}{ll}
G_{11}(\mathbf{k}, \omega), & G_{12}(\mathbf{k}, \omega) \\
G_{21}(\mathbf{k}, \omega), & G_{22}(\mathbf{k}, \omega)
\end{array}\right)
$$

is a convolution of the spin Green's function and charge carrier Green's function (6), and its diagonal and offdiagonal components $G_{11}\left(i-j, t-t^{\prime}\right)=\left\langle\left\langle C_{i \sigma}(t) ; C_{j \sigma}^{\dagger}\left(t^{\prime}\right)\right\rangle\right\rangle$ and $G_{21}\left(i-j, t-t^{\prime}\right)=\left\langle\left\langle C_{i \uparrow}^{\dagger}(t) ; C_{j \downarrow}^{\dagger}\left(t^{\prime}\right)\right\rangle\right\rangle$ have been obtained as $\frac{25}{2}$,

$$
\begin{aligned}
G_{11}(\mathbf{k}, \omega) & =\frac{1}{N} \sum_{\mathbf{p}} Z_{F} \frac{B_{\mathbf{p}}}{2 \omega_{\mathbf{p}}}\left\{\operatorname { c o t h } [ \frac { 1 } { 2 } \beta \omega _ { \mathbf { p } } ] \left(\frac{U_{h \mathbf{p}+\mathbf{k}}^{2}}{\omega+E_{h \mathbf{p}+\mathbf{k}}-\omega_{\mathbf{p}}}+\frac{U_{h \mathbf{p}+\mathbf{k}}^{2}}{\omega+E_{h \mathbf{p}+\mathbf{k}}+\omega_{\mathbf{p}}}\right.\right. \\
& \left.+\frac{V_{h \mathbf{p}+\mathbf{k}}^{2}}{\omega-E_{h \mathbf{p}+\mathbf{k}}+\omega_{\mathbf{p}}}+\frac{V_{h \mathbf{p}+\mathbf{k}}^{2}}{\omega-E_{h \mathbf{p}+\mathbf{k}}-\omega_{\mathbf{p}}}\right)+\tanh \left[\frac{1}{2} \beta E_{h \mathbf{p}+\mathbf{k}}\right]\left(\frac{U_{h \mathbf{p}+\mathbf{k}}^{2}}{\omega+E_{h \mathbf{p}+\mathbf{k}}+\omega_{\mathbf{p}}}\right. \\
& \left.\left.-\frac{U_{h \mathbf{p}+\mathbf{k}}^{2}}{\omega+E_{h \mathbf{p}+\mathbf{k}}-\omega_{\mathbf{p}}}+\frac{V_{h \mathbf{p}+\mathbf{k}}^{2}}{\omega-E_{h \mathbf{p}+\mathbf{k}}-\omega_{\mathbf{p}}}-\frac{V_{h \mathbf{p}+\mathbf{k}}^{2}}{\omega-E_{h \mathbf{p}+\mathbf{k}}+\omega_{\mathbf{p}}}\right)\right\}, \\
G_{21}(\mathbf{k}, \omega) & =\frac{1}{N} \sum_{\mathbf{p}} Z_{F} \frac{\bar{\Delta}_{h Z}(\mathbf{p}+\mathbf{k})}{2 E_{h \mathbf{p}+\mathbf{k}}} \frac{B_{\mathbf{p}}}{2 \omega_{\mathbf{p}}}\left\{\operatorname { c o t h } [ \frac { 1 } { 2 } \beta \omega _ { \mathbf { p } } ] \left(\frac{1}{\omega-E_{h \mathbf{p}+\mathbf{k}}-\omega_{\mathbf{p}}}+\frac{1}{\omega-E_{h \mathbf{p}+\mathbf{k}}+\omega_{\mathbf{p}}}\right.\right. \\
& \left.-\frac{1}{\omega+E_{h \mathbf{p}+\mathbf{k}}+\omega_{\mathbf{p}}}-\frac{1}{\omega+E_{h \mathbf{p}+\mathbf{k}}-\omega_{\mathbf{p}}}\right)+\tanh \left[\frac{1}{2} \beta E_{h \mathbf{p}+\mathbf{k}}\right]\left(\frac{1}{\omega-E_{h \mathbf{p}+\mathbf{k}}-\omega_{\mathbf{p}}}\right. \\
& \left.\left.-\frac{1}{\omega-E_{h \mathbf{p}+\mathbf{k}}+\omega_{\mathbf{p}}}-\frac{1}{\omega+E_{h \mathbf{p}+\mathbf{k}}+\omega_{\mathbf{p}}}+\frac{1}{\omega+E_{h \mathbf{p}+\mathbf{k}}-\omega_{\mathbf{p}}}\right)\right\},
\end{aligned}
$$

respectively, where the electron quasiparticle coherent weight $Z_{F}=Z_{h F} / 2$, and the spin excitation spectrum $\omega_{\mathbf{p}}$ and $B_{\mathbf{p}}$ have been given in Ref. 25 .

In the presence of a single point-like impurity scattering potential,

$$
\tilde{V}=V_{0} \delta(\mathbf{r}) \tau_{3},
$$

the unperturbed electron Green's function in Eq. (7) is dressed by this impurity scattering, where the $T$ matrix exactly accounts for multiple scattering off that impurity ${ }^{32}$. Since translational invariance is broken by the impurity, the dressed electron Green's function in the Nambu representation in real-space depends on two positions $\mathbf{r}$ and $\mathbf{r}^{\prime}$,

$$
\tilde{G}\left(\mathbf{r}, \mathbf{r}^{\prime}, \omega\right)=G\left(\mathbf{r}-\mathbf{r}^{\prime}, \omega\right)+G(\mathbf{r}, \omega) \tilde{T}(\omega) G\left(-\mathbf{r}^{\prime}, \omega\right),
$$

with the impurity induced $T$ matrix can be obtained as ${ }^{32}$,

$$
\tilde{T}(\omega)=\frac{1}{1-G(\omega) V_{0} \tau_{3}} V_{0} \tau_{3},
$$

where $G(\omega)=(1 / N) \sum_{\mathbf{k}} G(\mathbf{k}, \omega)$. In this case, LDOS of the system can be obtained as,

$$
\rho(\mathbf{r}, \omega)=-\frac{1}{\pi} \operatorname{Im} \tilde{G}(\mathbf{r}, \omega)=\rho_{0}(\omega)+\delta \rho(\mathbf{r}, \omega),
$$

where the homogeneous density of states $\rho_{0}(\omega)=$ $-\operatorname{Im} G(0, \omega) / \pi$, and is uniform in real-space, therefore it reflects a homogenous background, while $\delta \rho(\mathbf{r}, \omega)$ is the modulation for the homogeneous density of states due to the presence of the impurity scattering potential (9), and can be obtained as,

$$
\begin{aligned}
\delta \rho(\mathbf{r}, \omega) & =\rho(\mathbf{r}, \omega)-\rho_{0}(\omega) \\
& =-\frac{1}{\pi} \operatorname{Im}[G(\mathbf{r}, \omega) \tilde{T}(\omega) G(-\mathbf{r}, \omega)]_{11},
\end{aligned}
$$

and its Fourier transform is evaluated explicitly as,

$\delta \rho(\mathbf{q}, \omega)=-\frac{1}{\pi} \operatorname{Im}\left(\frac{1}{N} \sum_{\mathbf{k}}[G(\mathbf{k}+\mathbf{q}, \omega) \tilde{T}(\omega) G(\mathbf{k}, \omega)]_{11}\right)$. 
This LDOS is closely related to the differential conductance $g(\mathbf{r}, \omega)$ since the result of the differential conductance $g(\mathbf{r}, \omega)$ is proportional to $\rho(\mathbf{r}, \omega)$ at location $\mathbf{r}$ and energy $\omega=\mathrm{eV}$. However, the intense atomic-scale spatial fluctuations in electronic structure cause systematic errors in setting the STM tip elevation for the experimental measurement of the differential conductance $g(\mathbf{r}, \omega)^{12-14}$, therefore the ratio of differential conductances at opposite bias in Eq. (11) or its equivalent, the ratio of LDOS at opposite energy,

$$
Z(\mathbf{r}, \omega)=\frac{\rho(\mathbf{r}, \omega)}{\rho(\mathbf{r},-\omega)},
$$

has been measured experimentally for an enhancement of the QSI signatures ${ }^{12-14}$. Since the condition $\rho_{0}(\omega) \gg$ $\delta \rho(\mathbf{r}, \omega)$ is well satisfied for cuprate superconductors, then the ratio of LDOS at opposite energy (15) can be obtained approximately as ${ }^{33}$,

$$
Z(\mathbf{r}, \omega) \approx Z_{0}(\omega)\left[1+\frac{\delta \rho(\mathbf{r}, \omega)}{\rho_{0}(\omega)}-\frac{\delta \rho(\mathbf{r},-\omega)}{\rho_{0}(-\omega)}\right]
$$

where $Z_{0}(\omega)=\rho_{0}(\omega) / \rho_{0}(-\omega)$, and only the first-order modulation $\delta \rho(\mathbf{r}, \pm \omega)$ is kept, then the modulation of the ratio of LDOS at opposite energy can be expressed as,

$$
\begin{aligned}
\delta Z(\mathbf{r}, \omega) & =Z(\mathbf{r}, \omega)-Z_{0}(\omega) \\
& \approx Z_{0}(\omega)\left[\frac{\delta \rho(\mathbf{r}, \omega)}{\rho_{0}(\omega)}-\frac{\delta \rho(\mathbf{r},-\omega)}{\rho_{0}(-\omega)}\right]
\end{aligned}
$$

and its Fourier transformation is evaluated explicitly as,

$$
\delta Z(\mathbf{q}, \omega) \approx Z_{0}(\omega)\left[\frac{\delta \rho(\mathbf{q}, \omega)}{\rho_{0}(\omega)}-\frac{\delta \rho(\mathbf{q},-\omega)}{\rho_{0}(-\omega)}\right] .
$$

It has been argued ${ }^{12-14}$ that the observed QSI from $\delta Z(\mathbf{r}, \omega)$ or $\delta Z(\mathbf{q}, \omega)$ is an intrinsic phenomenon free from any set-point-related issues, which inevitably contaminate $g(\mathbf{r}, \omega)$ and $g(\mathbf{q}, \omega)$.

We are now ready to discuss the QSI phenomenon in cuprate superconductors in the SC state and its extinction at the high energy. In cuprate superconductors, although the values of $J$ and $t$ is believed to vary somewhat from compound to compound, however, as a qualitative discussion, the commonly used parameters in this paper are chosen as $t / J=2.5$ and $t^{\prime} / t=0.3$. In this case, we have performed a calculation for the Fourier transformed LDOS $\delta \rho(\mathbf{q}, \omega)$ in Eq. (14) and the Fourier transformed ratio of LDOS at opposite energy $\delta Z(\mathbf{q}, \omega)$ in Eq. (18), and the results of $|\delta \rho(\mathbf{q}, \omega)|$ as a function of momentum in the full Brillouin zone with energy (a) $\omega=-0.01 \mathrm{~J}$, (b) $\omega=-0.03 J$, and (c) $\omega=-0.05 J$ at temperature $T=0.002 J$ for the doping concentration $\delta=0.15$ in the presence of single point-like potential scatterer of strength $V=0.1 J$ are plotted in Fig. 1 (left panel). For comparison, the corresponding results of $|\delta Z(\mathbf{q}, \omega)|$ are also plotted in Fig. 1d-f (right panel). It is shown that the results obtained from $\delta Z(\mathbf{q}, \omega)$ are almost the same as those of $\delta \rho(\mathbf{q}, \omega)$, reflecting an experimental fact that the measurement data from the ratio of LDOS at opposite energy retain all the main information of QSI observed from LDOS. Moreover, both results from $\delta Z(\mathbf{q}, \omega)$
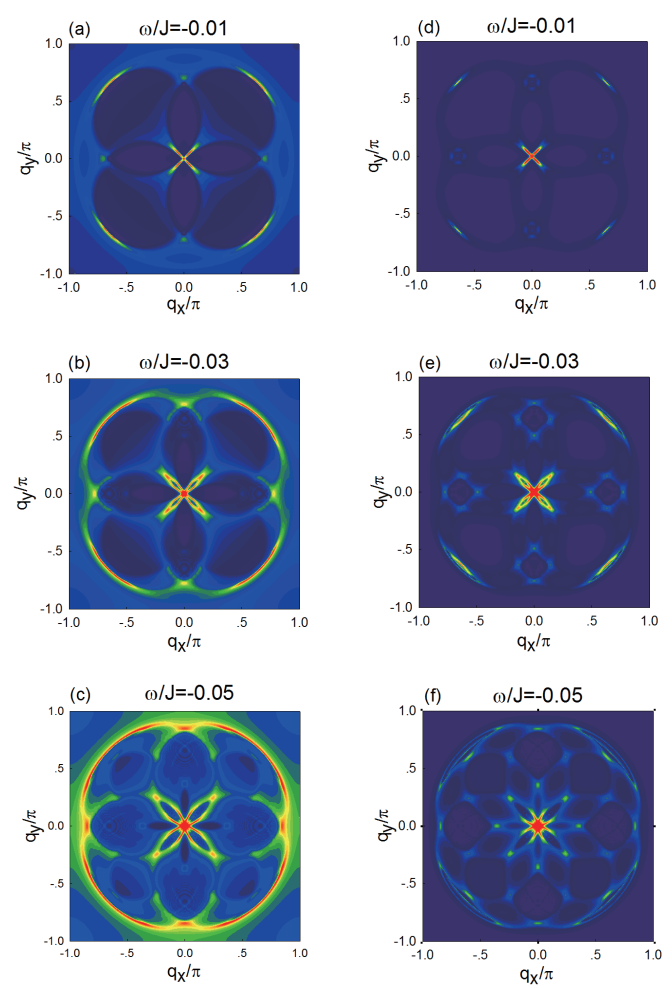

FIG. 1: (Color online) The Fourier transformed LDOS as a function of momentum in the full Brillouin zone with energy (a) $\omega=-0.01 J$, (b) $\omega=-0.03 J$, and (c) $\omega=-0.05 J$ at temperature $T=0.002 J$ for the doping concentration $\delta=0.15$ in the presence of single point-like potential scatterer of strength $V=0.1 J$ (left panel). (d) to (f) are the corresponding results of the Fourier transformed ratio of LDOS at opposite energy (right panel).

and $\delta \rho(\mathbf{q}, \omega)$ are clearly fourfold symmetric and display numerous local maxima (bright regions) at different $\mathbf{q}$ for different energies, where all of these $\mathbf{q}$ vectors are consistent with the prediction from the octet model ${ }^{4,5}$. These bright regions in momentum-space display the intensity of the modulation for LDOS (Fig. 1a-c) or the intensity of the modulation for the ratio of LDOS at opposite energy (Fig. 1d-f). In particular, the bright regions near the center of each figure in Fig. 1 a-c or Fig. 1d-f reflect long-wavelength inhomogeneity in $\delta \rho(\mathbf{q}, \omega)$ or $\delta Z(\mathbf{q}, \omega)$, and are induced by the weak impurity scattering potential (9). All these results are in qualitative agreement with the STM experimental results ${ }^{4,5}$.

To analyze this peak feature in Fig. 1 more clearly, we have made a series of calculations for the momentum dependence of $\delta \rho(\mathbf{q}, \omega)$ and $\delta Z(\mathbf{q}, \omega)$ with different energies, and the results of $|\delta \rho(\mathbf{q}, \omega)|$ as a function of momentum along the (a) $[0,0] \rightarrow[\pi, \pi]$ (nodal) direction and (b) $[0,0] \rightarrow[\pi, 0]$ (antinodal) direction with energy $\omega=-0.01 J, \omega=-0.02 J, \omega=-0.03 J, \omega=-0.04 J$, and $\omega=-0.05 J$ (from bottom to top) at temperature $T=0.002 J$ for the doping concentration $\delta=0.15$ in the presence of single point-like potential scatterer of strength $V=0.1 J$ are plotted in Fig. 2 (left panel). For comparison, the corresponding results of $|\delta Z(\mathbf{q}, \omega)|$ 

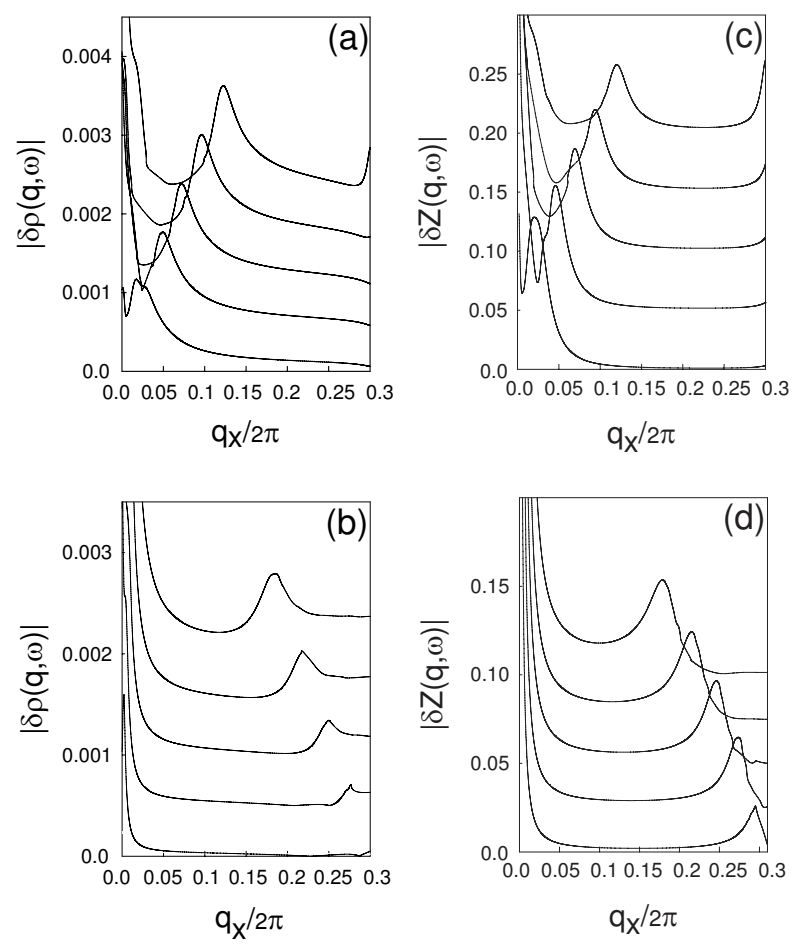

FIG. 2: The Fourier transformed LDOS as a function of momentum along the (a) $[0,0] \rightarrow[\pi, \pi]$ direction and (b) $[0,0] \rightarrow[\pi, 0]$ direction with energy $\omega=-0.01 J, \omega=-0.02 J$, $\omega=-0.03 J, \omega=-0.04 J$, and $\omega=-0.05 J$ (from bottom to top) at temperature $T=0.002 J$ for the doping concentration $\delta=0.15$ in the presence of single point-like potential scatterer of strength $V=0.1 J$ (left panel). (c) and (d) are the corresponding results of the Fourier transformed ratio of LDOS at opposite energy (right panel).
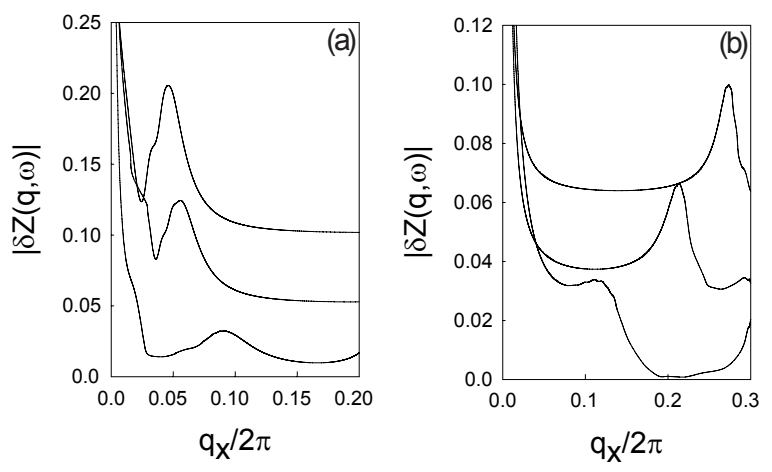

FIG. 3: The Fourier transformed ratio of LDOS at opposite energy as a function of momentum along the (a) $[0,0] \rightarrow$ $[\pi, \pi]$ direction and (b) $[0,0] \rightarrow[\pi, 0]$ direction with energy $\omega=-0.02 J$ at temperature $T=0.002 J$ for the doping concentration $\delta=0.08, \delta=0.11$, and $\delta=0.15$ (from bottom to top) in the presence of single point-like potential scatterer of strength $V=0.1 J$.

are also plotted in Fig. 2c-d (right panel). In corresponding to the results in Fig. 1, the results in Fig. 2 show that although the peak in $\delta \rho(\mathbf{q}, \omega)$ (then the intensity of the modulation for LDOS) or the peak in $\delta Z(\mathbf{q}, \omega)$ (then the intensity of the modulation for the ratio of LDOS at opposite energy) is changing rapidly and intricately with energy, the peaks, whose q-vectors oriented towards the antinodal direction, appear at finite $|\mathbf{q}|$ at very low energy and then move steadily inwards toward the center, while the peaks with $\mathbf{q}$-vectors along the nodal direction, appear and move steadily to large $|\mathbf{q}|$ with increasing energy, in qualitative agreement with the STM experimental results.5. However, although the position of the peak obtained from $\delta \rho(\mathbf{q}, \omega)$ is almost the same as that from $\delta Z(\mathbf{q}, \omega)$, the peak intensity obtained from $\delta Z(\mathbf{q}, \omega)$ is much stronger than that appeared in $\delta \rho(\mathbf{q}, \omega)$, and therefore there is an enhancement of the QSI signatures in $\delta Z(\mathbf{q}, \omega)$. This expected difference of the peak intensity obtained from $\delta \rho(\mathbf{q}, \omega)$ and $\delta Z(\mathbf{q}, \omega)$ can be understood from the definition of $\delta Z(\mathbf{q}, \omega)$ in Eq. (18). Although the modulations in $\delta \rho(\mathbf{r}, \omega)$ and $\delta \rho(\mathbf{r},-\omega)$ are spatially quite similar, the spatial-phase relation for QSI is not known precisely. However, $\delta Z(\mathbf{q}, \omega)$ is sensitive to the relation signs between $\delta \rho(\mathbf{q}, \omega)$ and $\delta \rho(\mathbf{q},-\omega)$, namely, taking the ratio of LDOS reduces the in-plane component $\underline{13}$. This is why the the QSI extinction at the high energy in cuprate superconductors in the SC state is firstly observed from the experimental measurement of $\delta Z(\mathbf{q}, \omega) \underline{12}-14$.

For a better understanding of the physical properties of the QSI phenomenon in cuprate superconductors, we have further performed a calculation for $\delta Z(\mathbf{q}, \omega)$ with different doping concentrations, and the results of $|\delta Z(\mathbf{q}, \omega)|$ as a function of momentum along the (a) $[0,0] \rightarrow[\pi, \pi]$ (nodal) direction and (b) $[0,0] \rightarrow[\pi, 0]$ (antinodal) direction with energy $\omega=-0.02 J$ at temperature $T=0.002 J$ for the doping concentration $\delta=0.08$, $\delta=0.11$, and $\delta=0.15$ (from bottom to top) in the presence of single point-like potential scatterer of strength $V=0.1 J$ are plotted in Fig. 3. Obviously, the peak is doping dependent. With increasing the doping concentration, the weight of the peak increases. Furthermore, the position of the peak along the nodal direction appears at finite $|\mathbf{q}|$ in the low doping concentration, and then moves towards to the center of the Brillouin zone with increasing doping concentration. In contrast to the case along the nodal direction, the position of the peak along the antinodal direction is located at finite $|\mathbf{q}|$ in the low doping concentration, and then is shifted to large $|\mathbf{q}|$ with increasing doping concentration.

The energy dependence of the peaks in Fig. 2 has been used to extract the dispersion of the peaks, and the results of (a) the positions and (b) amplitudes of the lowest energy peaks in $|\delta Z(\mathbf{q}, \omega)|$ as a function of energy along the $[0,0] \rightarrow[\pi, \pi]$ direction for temperature $T=0.002 J$ at the doping concentration $\delta=0.15$ in the presence of single point-like potential scatterer of strength $V=0.1 \mathrm{~J}$ are plotted in Fig. 4. For comparison, the corresponding STM experimental results 14 of cuprate superconductors are also shown in Fig. 4 (inset). Our results show clearly that the sharp peak persists in a large momentum space region along the nodal direction and are in qualitative agreement with the STM experimental result $\stackrel{14}{ }$. However, the amplitude of the peak increases with increasing energy at the low energy, and reaches a maximum at the intermediate energy, then decreases rapidly with further increasing energy. Apparently, there is a substantial difference between theory and experiment, namely, the amplitude of the peak obtained from theory does not di- 


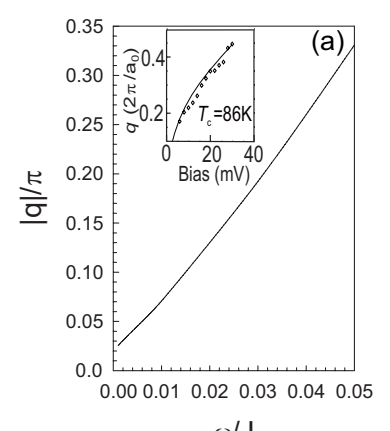

$\omega / \mathrm{J}$

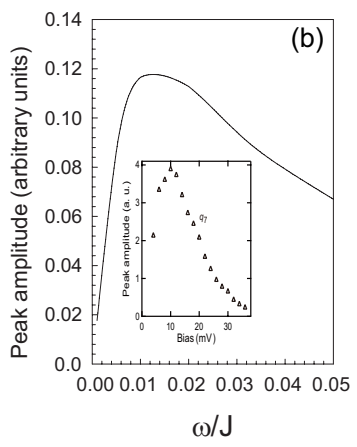

FIG. 4: (a) The positions and (b) amplitudes of the lowest energy peaks in the Fourier transformed ratio of LDOS at opposite energy as a function of energy along the $[0,0] \rightarrow[\pi, \pi]$ direction for temperature $T=0.002 J$ at the doping concentration $\delta=0.15$ in the presence of single point-like potential scatterer of strength $V=0.1 J$. Insets: the corresponding experimental results for cuprate superconductors taken from Ref. 14.

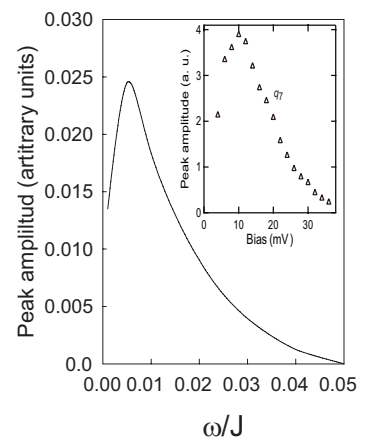

FIG. 5: The amplitudes of the lowest energy peaks in the Fourier transformed ratio of LDOS at opposite energy as a function of energy along the $[0,0] \rightarrow[\pi, \pi]$ direction with temperature $T=0.002 J$ at the doping concentration $\delta=0.15$ for parameters $\Gamma=0.005 \mathrm{~J}$ and $\alpha=0.2$ in the presence of single point-like potential scatterer of strength $V=0.1 J$. Inset: the corresponding experimental result for cuprate superconductors taken from Ref. 14 .

minish to zero at the high energy. This obvious weakness is a natural consequence of the neglect of the imaginary part of the self-energy (then the scattering rate). To obtain the full charge carrier Green function (6) within the kinetic energy driven SC mechanism, we $\underline{\underline{17}, 25}$ have made a self-consistent calculation in the static limit approximation for the real part of the charge carrier selfenergy $\operatorname{Re} \Sigma_{1}^{(h)}(\omega)$ induced by the interaction between the charge carriers and spins, where its antisymmetric part has been obtained as $Z_{h F}^{-1}=1-\left.\operatorname{Re} \Sigma_{1 o}^{(h)}(\mathbf{k}, \omega=0)\right|_{\mathbf{k}=[\pi, 0]}$ and therefore is closely related to the charge carrier quasiparticle coherent weight, while its symmetric part $\operatorname{Re} \Sigma_{1 e}^{(h)}(\mathbf{k}, \omega=0)$ is a constant around the Fermi surface, and it just renormalizes the chemical potential. However, the imaginary part of the charge carrier self-energy $\operatorname{Im} \Sigma_{1}^{(h)}(\mathbf{k}, \omega)$ has been dropped 17,25 . This leads to a fact that the imaginary part of the renormalized electron selfenergy in the dressed electron Green's function (10) due to the presence of the impurity scattering potential (9) has been neglected. In this case, for the present discussions of the QSI extinction at the high energy, we need to introduce an imaginary part of the electron self-energy (then the scattering rate) in the electron Green function as,

$$
i \operatorname{Im} \Sigma(\omega)=i(\Gamma+\alpha \omega),
$$

for a compensation due to the neglect of the imaginary part of the renormalized self-energy, where $\Gamma$ and $\alpha$ are constant. It has been argued ${ }^{34}$ that this type of the energy dependent inelastic scattering rate (19) seems to be an intrinsic property of the electronic structure of cuprate superconductors, since it has been used to provide a robust fit for the spatially inhomogeneous differential conductances of cuprate superconductor based on the phenomenological d-wave BCS formalism. By considering this scattering rate (19) in the electron Green function, we therefore find that the dispersing QSI always disappears at the high energy. To show this point clearly, we have made a series of calculations for $\delta Z(\mathbf{q}, \omega)$ with different momenta and energies, and the result of the amplitudes of the lowest energy peaks in $|\delta Z(\mathbf{q}, \omega)|$ as a function of energy along the $[0,0] \rightarrow[\pi, \pi]$ direction for temperature $T=0.002 \mathrm{~J}$ at the doping concentration $\delta=0.15$ for parameters $\Gamma=0.005 J$ and $\alpha=0.2$ in the presence of single point-like potential scatterer of strength $V=0.1 \mathrm{~J}$ is plotted in Fig. 5 in comparison with the corresponding STM experimental result $\frac{14}{14}$ of cuprate superconductors (inset). In comparison with the result in Fig. 4b, although the amplitude of the peak is severely suppressed due to the presence of the scattering rate (19), it diminishes to zero (then the QSI extinction) at the high energy. Moreover, during the calculations, we find that the constant scattering rate $\Gamma$ in Eq. (19) only plays a subsidiary role, while the QSI modulation is further weakened by the effective scattering rate $\alpha \omega$. The similar conclusion has been obtained in Ref. 34 where they also find that the effective scattering rate $\alpha \omega$ plays a key role. Using a reasonably estimative value of $J \approx 120 \sim 150$ $\mathrm{meV}$, the peak disappears around the energy $6 \mathrm{meV} \sim 7.5$ $\mathrm{meV}$. Apparently, there is still a substantial difference between theory and experiment, namely, the energy value of the QSI extinction calculated theoretically is smaller than the corresponding energy value measured in the experiment. However, upon a closer examination one can see immediately that the main difference is due to fact that the calculated peak energy decreases rapidly with energy at high energy. This energy value of the QSI extinction is also smaller than the corresponding value of the charge carrier pairing gap 25 . The simple $t-J$ model can not be regarded as a complete model for the quantitative comparison with cuprate superconductors, however, as for a qualitative discussion in this paper, the overall shape seen in the theoretical result is qualitatively consistent with that observed in the STM experiment 14 .

A nature question is why QSI below the high energy in cuprate superconductors and its extinction at the high energy can be described qualitatively in the framework of the kinetic energy driven $\mathrm{SC}$ mechanism in the presence of a single point-like impurity. This may be understood from the kinetic energy driven $\mathrm{SC}$ mechanism itsel $1 \mathrm{17}$. 
As we have mentioned above, in the framework of kinetic energy driven SC mechanism, the electron Cooper pairs originating from the $\mathrm{d}$-wave charge carrier pairing state are due to the charge-spin recombination, therefore there is a coexistence of the electron Cooper pair and short-range AF correlation, and hence the short-range $\mathrm{AF}$ fluctuation can persist into the SC state $\frac{17}{}$. In particular, this charge-spin recombination is characterized by a convolution of the spin Green's function and charge carrier Green's function 17 . The main consequence of this convolution is that the hole-like charge carrier d-wave BCS formalism is transferred into the electron-like dwave BCS formalism 25 . In other words, main difference between the hole-like charge carrier Green's function (6) and the electron-like Green's function (7) is a shift of the momentum by the $\operatorname{AF}$ wave vector $Q=[\pi, \pi]$ in the Brillouin zone $e^{25}$. This leads to a fact that the quasiparticle states near the antinodal points are broadened. Moreover, these quasiparticle states around the antinodal regime are further suppressed due to the presence of the impurity scattering potential (9). On the other hand, the kinetic energy driven d-wave SC state is controlled by both the SC gap function and the SC quasiparticle coherent weight, which indicates that only coherent Bogoliubov quasiparticles at the Fermi surface are available for superconductivity since everything happens at the Fermi surface. In this case, although the Bogoliubov quasiparticles can be excited by either positive or negative energies ${ }^{35}$, the excitation spectrum is a particle-hole symmetric thin Dirac cone around the nodal regime, then QSI below the high energy and its extinction at the high energy occur in this case, where this high energy which sets the QSI extinction is determined not only by the d-wave SC gap function alone but also by the coherent Bogoliubov quasiparticles located on the thin Dirac cone.

In summary, within the framework of the kinetic energy driven SC mechanism, we have discussed the QSI phenomenon in cuprate superconductors in the SC state by considering the single point-like impurity scattering potential. This QSI is characterized by the peaks in LDOS or the ratio of LDOS at opposite energy. By calculation of the momentum and energy dependence of the Fourier transformed LDOS $\delta \rho(\mathbf{q}, \omega)$ and the Fourier transformed ratio of LDOS at opposite energy $\delta Z(\mathbf{q}, \omega)$, we have shown that the remarkable QSI phenomenon observed from STM experiments on cuprate superconductors can be described qualitatively by a single point-like impurity in the kinetic energy driven homogeneous dwave SC state. The amplitude of the peak increases with increasing energy at the low energy, and reaches a maximum at the intermediate energy, then diminishes to zero at the high energy. The theory also predicts that with increasing doping concentration, the position of the peak along the nodal direction moves towards to the center of the Brillouin zone, while the position of the peak along the antinodal direction is shifted to large momentum region, which should be verified by further STM experiments.

Within the framework of the kinetic energy driven SC mechanism ${ }^{17}$, our present results of QSI below the high energy and its extinction at the high energy due to the presence of the impurity scattering potential (9) are qualitatively consistent with the recent STM experimental data $4.5,14$. Establishing this agreement is important to confirming the nature of a single impurity in the kinetic energy driven homogeneous d-wave SC state in cuprate superconductors. Although a quantitative understanding of the QSI phenomenon is not straightforward to obtain, and depends rather sensitively on the nature of the scattering medium, in this paper we are primarily interested in exploring the general notion of the QSI phenomenon induced by a single impurity in the kinetic energy driven homogeneous d-wave SC state. The qualitative agreement between the present theoretical results and STM experimental data also show that the presence of impurities plays a crucial role for the QSI phenomenon in cuprate superconductors in the SC state. Although this QSI extinction at the high energy 14 also can be fitted by using a phenomenological d-wave BCS formalism with the same imaginary part of the self-energy (19), however, no explicit calculations of the doping dependence of $|\delta Z(\mathbf{q}, \omega)|$, as shown in Fig. 3, can be made within a phenomenological d-wave BCS formalism.

Under the kinetic energy driven SC mechanism, the external magnetic field aligns the spins of the unpaired electrons, then the singlet charge carrier pairs can not take advantage of the lower energy offered by a spinpolarized state. In this case, the magnetic field dependence of the penetration depth and superfluid density have been studied recently $\stackrel{36}{ }$, where the superfluid density decreases with increasing magnetic field, in agreement with the experimental results $\mathrm{37}^{37}$. With this study $\underline{36}$, we therefore can predict that QSI should be magnetic field dependent, and then the energy value of the QSI extinction decreases with increasing magnetic field. One of the typical features of d-wave superconductivity in cuprate superconductors is the particle-hole symmetric octet of dispersive Bogoliubov quasiparticle interference modulations ${ }^{3}-\underline{5}$. However, the recent STM experiments ${ }^{38}$ have reported on this octet's evolution from low temperatures to well into the underdoped pseudogap regime, where no pronounced changes occur in the octet phenomenology at the superconductor's critical temperature $T_{c}$, and it survives up to at least temperature $T \sim 1.5 T_{c}$. Thus an important issue is how this octet's evolution from the SC state into the pseudogap regime is fitted within the framework of the kinetic energy driven superconductivity. These and the related issues are under investigation now.

\section{Acknowledgments}

The authors would like to thank H. S. Zhao for the helpful discussions. This work was supported by the National Natural Science Foundation of China under Grant No. 10774015, and the funds from the Ministry of Science and Technology of China under Grant Nos. 2006CB601002 and 2006CB921300. 
* To whom correspondence should be addressed, E-mail: spfeng@bnu.edu.cn

2 See, e.g., the review, Øystein Fischer, Martin Kugler, Ivan Maggio-Aprile, Christophe Berthod, and Christoph Renner, Rev. Mod. Phys. 79 (2007) 353-419.

3 S. H. Pan, J. P. O'Neal, R. L. Badzey, C. Chamon, H. Ding, J. R. Engelbrecht, Z. Wang, H. Eisaki, S. Uchida, A. K. Gupta, K.-W. Ng, E. W. Hudson, K. M. Lang and J. C. Davis, Nature 413 (2001) 282-285.

4 J. E. Hoffman, K. McElroy, D.-H. Lee, K. M. Lang, H. Eisaki, S. Uchida, and J. C. Davis, Science 297 (2002) 1148-1151.

${ }^{5}$ K. McElroy, R. W. Simmonds, J. E. Hoffman, D.-H. Lee, J. Orenstein, H. Eisaki, S. Uchida, and J. C. Davis, Nature 422 (2003) 592-596.

6 Q.-H. Wang and D.-H. Lee, Phys. Rev. B 67 (2003) 020511.

7 D. Zhang and C. S. Ting, Phys. Rev. B 67 (2003) 100506.

${ }^{8}$ L. Capriotti, D. J. Scalapino, and R. D. Sedgewick, Phys. Rev. B 68 (2003) 014508.

9 T. Pereg-Barnea and M. Franz, Phys. Rev. B 68 (2003) 180506(R).

${ }^{10}$ L.-Y. Zhu, W. A. Atkinson, and P. J. Hirschfeld, Phys. Rev. B 69 (2004) 060503; T. S. Nunner, W. Chen, B. M. Andersen, A. Melikyan, and P. J. Hirschfeld, Phys. Rev. B 73 (2006) 104511.

11 S. Misra, M. Vershinin, P. Phillips, and A. Yazdani, Phys. Rev. B 70 (2004) 220503(R).

12 Y. Kohsaka, C. Taylor, K. Fujita, A. Schmidt, C. Lupien, T. Hanaguri, M. Azuma, M. Takano, H. Eisaki, H. Takagi, S. Uchida, and J. C. Davis, Science 315 (2007) 1380-1385.

13 T. Hanaguri, Y. Kohsaka, J. C. Davis, C. Lupien, I. Yamada, M. Azuma, M. Takano, K. Ohishi, M. Ono, and H. Takagi, Nature Phys. 3 (2007) 865-871.

14 Y. Kohsaka, C. Taylor, P. Wahl, A. Schmidt, J. Lee, K. Fujita, J. W. Alldredge, K. McElroy, J. Lee, H. Eisaki, S. Uchida, D.-H. Lee, and J. C. Davis, Nature 454 (2008) 1072-1078.

15 Brian M. Anderson and P. J. Hirschfeld, Phys. Rev. B 79 (2009) 144515.

16 W. A. Atkinson, Phys. Rev. B 71 (2005) 024516.

17 Shiping Feng, Phys. Rev. B 68 (2003) 184501; Shiping Feng, Tianxing Ma, and Huaiming Guo, Physica C 436 (2006) 14-24.

18 See, e.g., the review, A. Damascelli, Z. Hussain, and Z. X. Shen, Rev. Mod. Phys. 75 (2003) 473-541, and references therein.

19 P. W. Anderson, Science 235 (1987) 1196-1198.

20 Shiping Feng, Jihong Qin, and Tianxing Ma, J. Phys. Condens. Matter 16 (2004) 343-359 (2004); Shiping Feng, Tianxing Ma, and Jihong Qin, Mod. Phys. Lett. B 17 (2003) 361-373.

21 See, e.g., the review, Shiping Feng, Huaiming Guo, Yu Lan, and Li Cheng, Int. J. Mod. Phys. B 22 (2008) 3757-3811.

22 R. B. Laughlin, Phys. Rev. Lett. 79 (1997) 1726-1729.
23 See, e.g., the review, Patrick A. Lee, Naoto Nagaosa, and Xiao-Gang Wen, Rev. Mod. Phys. 78 (2006) 17-85.

24 See, e.g., the review, L. Yu, in Recent Progress in ManyBody Theories, vol. 3, T. L. Ainsworth, C. E. Campbell, B. E. Clenceats, and E. Krotscheck, eds.( Plenum, 1992) P.157.

25 Huaiming Guo and Shiping Feng, Phys. Lett. A 361 (2007) 382-390; Yu Lan, Jihong Qin, and Shiping Feng, Phys. Rev. B 76 (2007) 014533; Zhi Wang and Shiping Feng, Phys. Rev. B 80 (2009) 064510.

26 Li Cheng and Shiping Feng, Phys. Rev. B 77 (2008) 054518; Shiping Feng, Tianxing $\mathrm{Ma}$, and Xintian Wu, Phys. Lett. A 352 (2006) 438-445.

27 Zhi Wang, Huaiming Guo, and Shiping Feng, Physica C 468 (2008) 1078; Zhi Wang and Shiping Feng, Phys. Rev. B 80 (2009) 174507.

28 P. Dai, H.A. Mook, R.D. Hunt, and F. Dog̃an, Phys. Rev. B 63 (2001) 054525; Ph. Bourges, B. Keimer, S. Pailhés, L.P. Regnault, Y. Sidis, and C. Ulrich, Physica C $\mathbf{4 2 4}$ (2005) 45.

29 M. Arai, T. Nishijima, Y. Endoh, T. Egami, S. Tajima, K. Tomimoto, Y. Shiohara, M. Takahashi, A. Garret, and S.M. Bennington, Phys. Rev. Lett. 83 (1999) 608; S.M. Hayden, H.A. Mook, P. Dai, T.G. Perring, and F. Dog̃an, Nature 429 (2004) 531; C. Stock, W.J.L. Buyers, R.A. Cowley, P.S. Clegg, R. Coldea, C.D. Frost, R. Liang, D. Peets, D. Bonn, W.N. Hardy, and R.J. Birgeneau, Phys. Rev. B 71 (2005) 024522.

${ }^{30}$ R. Harris, P. J. Turner, Saeid Kamal, A. R. Hosseini, P. Dosanjh, G. K. Mullins, J. S. Bobowski, C. P. Bidinosti, D. M. Broun, Ruixing Liang, W. N. Hardy, and D. A. Bonn, Phys. Rev. B 74 (2006) 104508.

${ }^{31}$ Leon Balents, Phys. Rev. B 62 (2000) 2346.

32 A. V. Balatsky, I. Vekhter, and Jian-Xin Zhu, Rev. Mod. Phys. 78 (2006) 373-433.

33 T. Pereg-Barnea and M. Franz, Phys. Rev. B 78 (2008) $020509(\mathrm{R})$.

34 J. W. Alldredge, Jinho Lee, K. McElroy, M. Wang, K. Fujita, Y. Kohsaka, C. Taylor, H. Eisaki, S. Uchida, P. J. Hirschfeld, and J. C. Davis, Nature Phys. 4 (2008) 319-326.

35 H. Matsui, T. Sato, T. Takahashi, S. C. Wang, H. B. Yang, H. Ding, T. Fujii, T. Watanabe, and A. Matsuda, Phys. Rev. Lett. 90 (2003) 217002; Alexander V. Balatsky, W. S. Lee, and Z. X. Shen, arXiv:0807.1893: Weifang Wang, Zhi Wang, Jingge Zhang, and Shiping Feng, Phys. Lett. A 374 (2010) 632-636.

36 Zheyu Huang, Huaisong Zhao, and Shiping Feng, unpublished.

37 J. E. Sonier, J. H. Brewer, R. F. Kiefl, G. D. Morris, R. I. Miller, D. A. Bonn, J. Chakhalian, R. H. Heffner, W. N. Hardy, and R. Liang, Phys. Rev. Lett. 83 (1999) 4156.

38 Jhinhwan Lee, K. Fujita, A. R. Schmidt, Chung Koo Kim, H. Eisaki, S. Uchida, and J. C. Davis, Science 325 (2009) 1099-1103. 